\title{
Local management of the nonlinearity of Bose-Einstein condensates with pinched potentials
}

\author{
A. Guerreiro and Nuno A. Silva* \\ Departamento de Física e Astronomia da Faculdade de Ciências da Universidade do Porto, Rua do Campo Alegre 687, \\ 4169-007 Porto, Portugal \\ and INESC TEC, Centre of Applied Photonics, Rua do Campo Alegre 687, 4169-007 Porto, Portugal
}

(Received 4 July 2016; published 5 December 2016)

\begin{abstract}
We present a proposal for the local control of the nonlinearity in quasi-one-dimensional Bose-Einstein condensates induced by a local pinching of the transverse confining potential. We investigate the scattering of bright matter-wave solitons through a pinched potential using numerical simulations of the full three-dimensional Gross-Pitaevskii equation and the corresponding effective one-dimensional model with spatially varying nonlinearity.
\end{abstract}

DOI: 10.1103/PhysRevA.94.063602

\section{INTRODUCTION}

Local and temporal management of nonlinearities is emerging for the manipulation of soliton dynamics [1]. For the case of localized matter-wave solitons in Bose-Einstein condensates (BECs), this is closely related to the control of the atomic interactions and constitutes an important tool in the exploration of macroscopic quantum-mechanical effects and the development of future quantum-enhanced technologies [2]. Temporal management has been explored for a myriad of effects, from generation and stabilization of bright solitons in high dimensional systems [3-5], to the creation of matterwave breathers [6-8], periodic matter waves [9], and shock waves [10]. Yet, much of the research in controlling soliton dynamics has been focused on exploiting the local management of the nonlinearity. In fact, there has been a wide variety of unusual nonlinear phenomena reported in these so-called collisionally inhomogeneous media, which include adiabatic compression of matter waves [11], atomic soliton lasers [12-14], enhanced transmission of matter waves through a potential barrier $[15,16]$, observation and control of Faraday waves [17], Bloch oscillations and dynamical trapping of matter wave solitons [18-20], and even the investigations of acoustic black holes and Hawking radiation [21-23], just to name a few. Therefore, for the experimental realization of these phenomena, it is of paramount importance to develop techniques that provide spatial management of the nonlinearity in BECs.

The standard approach to local management of the nonlinearity in BECs is the localized control of the interactions through the Feshbach resonance management (FRM). Conceptually, it consists in using an external control field (such as a magnetic [24] or an optical [25] field) to vary the atomic scattering length in the vicinity of the Feshbach resonance. Unfortunately for many interesting experiments involving spatial varying nonlinearities, both magnetic and optical FRM have proven to be unsuitable: the former because the length scale of the variations of the magnetic field is much larger than the size of the condensate, which strongly limits the experimental applications, and the latter because, in addition to a reduction of the lifetime of the quantum gas, a parasitic dipole force potential is produced that typically dominates the dynamics [26].

\footnotetext{
*Corresponding author: nunoazevedosilva@gmail.com
}

An alternative approach is available for quasi-onedimensional (1D) settings, where the BEC is tightly confined in a cigar-shaped trap. In such systems, one can exploit the dependence of the effective nonlinearity on the strength of the confinement potential to mimic both a temporal [11] and a spatial $[27,28]$ management of the nonlinearity. In the case of spatial management, a variation of the confinement potential results in a spatial modulation of an additional effective linear potential, which typically dominates the dynamics of the solitons [28].

In this work, we propose a strategy for the local management of the nonlinearity in quasi-1D systems based on a pinch of the confinement potential. First, in Sec. II, we introduce an effective model for the quasi-1D BEC and demonstrate how a specific anisotropic variation of the transverse trapping potential along the longitudinal direction allows us to control independently the effective linear and nonlinear parameters of the model. In Sec. III, we further explore the effect of a pinch of the potential in terms of the transverse dynamics of the BEC, comparing the strategy with the standard FRM techniques and advancing a proposal for an experimental realization of such traps. In Sec. IV we study the dynamics of the scattering of a bright soliton through a pinched potential, comparing the numerical results obtained for full three-dimensional Gross-Pitaevskii equation and of the corresponding effective one-dimensional model, which are found to be in very good agreement. Finally in Sec. V we present our conclusions and discuss future directions of research.

\section{MODEL}

In the mean-field approximation, the dynamics of a BEC tightly confined in the transverse $x y$ plane by an external harmonic potential can be described by a macroscopic wave function $\Psi(\boldsymbol{r}, t)$, obeying the Gross-Pitaevskii equation (GPE)

$$
i \hbar \partial_{t} \Psi+\frac{\hbar^{2}}{2 m} \nabla^{2} \Psi-V_{\text {ext }}(\mathbf{r}) \Psi+g|\Psi|^{2} \Psi=0,
$$

such that $\int d \boldsymbol{r}|\Psi(\boldsymbol{r}, t)|^{2}=N_{a}$ is the number of atoms. Also, $g=4 \pi \hbar^{2} a_{s} / m$ is the nonlinear constant in terms of the atomic mass $m$ and scattering length $a_{s}$. This work considers an external trapping potential of the form $V_{\text {ext }}(\mathbf{r})=$ $m\left[\omega_{x}^{2} x^{2}+\omega_{y}^{2} y^{2}\right] / 2$ with frequencies $\omega_{x}=\omega_{x}(z)$ and $\omega_{y}=$ $\omega_{y}(z)$ that vary along the longitudinal direction $z$. In this 
setting, provided that the confinement potential is sufficiently strong, the kinetic energy in the transverse directions typically governs over interatomic interaction and Eq. (1) can be reduced to an effective quasi-1D model. This paper follows the approach described in Refs. [29,30], although alternative approaches can be found in the literature with wide validity ranges [27,28]. For slowly varying $\omega_{x}$ and $\omega_{y}$ along $z$, the ansatz solution $\Psi \equiv \psi(x, y, z, t)=\varphi(z, t) \chi(x, y, z)$ can be used, where $\varphi(z, t)$ and $\chi(x, y, z)$ describe respectively the fast longitudinal and slow transverse dynamics (corresponding to $\partial_{z}^{2} \chi \approx 0$ ). Then, Eq. (1) can be recast into two coupled equations:

$$
\begin{gathered}
i \hbar \partial_{t} \varphi=-\frac{\hbar^{2}}{2 m} \partial_{z}^{2} \varphi+\bar{\mu} \varphi \\
\bar{\mu} \chi=-\frac{\hbar^{2}}{2 m} \nabla_{\perp}^{2} \chi+\frac{m}{2}\left[\omega_{x}^{2} x^{2}+\omega_{y}^{2} y^{2}\right] \chi-g|\varphi|^{2}|\chi|^{2} \chi .
\end{gathered}
$$

For small values of the nonlinear term $g|\varphi|^{2}$, the chemical potential $\bar{\mu}$ is obtained by perturbative methods [29,31]. Taking the transverse wave function $\chi$ close to the ground state of the two-dimensional (2D) anisotropic harmonic potential $\quad \chi_{0}(x, y, z)=\sqrt{m /(\hbar \pi)}\left(\omega_{x} \omega_{y}\right)^{1 / 4} \exp \left[-m\left(\omega_{x} x^{2}+\right.\right.$ $\left.\left.\omega_{y} y^{2}\right) /(2 \hbar)\right]$, the perturbative expansion of the chemical potential to the lowest order of the density $|\varphi|^{2}$ is given by

$$
\bar{\mu} \approx \bar{\mu}_{0}-\mu_{\mathrm{nl}}=\bar{\mu}_{0}-g \frac{m}{2 \pi \hbar} \sqrt{\omega_{x} \omega_{y}}|\varphi|^{2},
$$

with $\bar{\mu}_{0}=\hbar\left[\omega_{x}+\omega_{y}\right] / 2$ and the first-order correction term computed from $\mu_{\mathrm{nl}}=g|\varphi|^{2} \int\left|\chi_{0}\right|^{4} d x d y$. Direct substitution of $\bar{\mu}$ into Eq. (2) yields the reduced 1D GPE

$$
i \hbar \partial_{t} \varphi+\frac{\hbar^{2}}{2 m} \partial_{z}^{2} \varphi-V_{\mathrm{eff}}(z) \varphi+g_{\mathrm{eff}}(z)|\varphi|^{2} \varphi=0,
$$

where $\quad V_{\text {eff }}(z) \equiv \hbar\left[\omega_{x}(z)+\omega_{y}(z)\right] / 2 \quad$ and $\quad g_{\text {eff }}(z) \equiv$ $[\mathrm{gm} /(2 \pi \hbar)] \sqrt{\omega_{x}(z) \omega_{y}(z)}$ are the effective linear potential and the nonlinear coefficient, respectively. Thus, the longitudinal variation of the confinement potential results in a spatial variation of both the effective linear potential and nonlinear coefficient of the reduced 1D model, which resembles the results of Refs. [27] and [28]. However, our approach allows us to control $g_{\text {eff }}$ and $V_{\text {eff }}$ independently by considering distinct $\omega_{x}$ and $\omega_{y}$. In particular, imposing

$$
\omega_{x}(z)=\bar{\omega}+\Delta \omega(z) \text { and } \omega_{y}(z)=\bar{\omega}-\Delta \omega(z)
$$

provides a practical way of producing spatial modulations of the effective nonlinear coefficient $g_{\text {eff }}(z) \equiv$ $[\mathrm{gm} /(2 \pi \hbar)] \sqrt{\bar{\omega}^{2}-\Delta \omega(z)^{2}}$ that keep the effective potential $V_{\text {eff }}=\hbar \bar{\omega}$ constant along $z$. Under these conditions, the transformation $\varphi \rightarrow \exp (-i \bar{\omega} t) \varphi$ followed by the normalizations $t \rightarrow \bar{\omega} t, z \rightarrow z / \sqrt{\hbar / \bar{\omega} m}$, and $\varphi \rightarrow \sqrt{2 \pi \hbar /(g m)} \varphi$ recast Eq. (5) as

$$
i \partial_{t} \varphi+\frac{1}{2} \partial_{z}^{2} \varphi+\tilde{g}_{\mathrm{eff}}(z)|\varphi|^{2} \varphi=0
$$

with a normalized nonlinearity given by $\tilde{g}_{\text {eff }}(z)=$ $\sqrt{1-[\Delta \omega(z) / \bar{\omega}]^{2}}$. This approach captures many features of the original 3D GPE, including the conservation of the total mass $\mathcal{N}$ and hamiltonian $\mathcal{H}$, given by

$$
\begin{gathered}
\mathcal{N}=\int_{-\infty}^{+\infty}|\varphi|^{2} d z \\
\mathcal{H}=\frac{1}{2} \int_{-\infty}^{+\infty}\left[\left|\partial_{z} \varphi\right|^{2}-\frac{\tilde{g}_{\text {eff }}(z)}{2}|\varphi|^{4}\right] d z .
\end{gathered}
$$

The main limitations of the effective 1D model arise from the two approximations used, namely the assumptions of slow transverse dynamics and the small nonlinear term.

The assumption of slow transverse dynamics is valid as long as the contribution to the chemical potential $\bar{\mu}$ from the terms proportional to $\partial_{z}^{2} \chi$ and $\partial_{z} \chi \partial_{z} \varphi / \varphi$ (which have been neglected) are small when compared to the nonlinear term. The contribution to the chemical potential from these terms, $\mu_{\text {slow }}$, calculated using perturbation theory to the first order is

$$
\mu_{\text {slow }}=\frac{\left(\partial_{z} \omega_{x}\right)^{2}}{8 \omega_{x}^{2}}+\frac{\left(\partial_{z} \omega_{y}\right)^{2}}{8 \omega_{y}^{2}}
$$

which can be neglected as long as

$$
\frac{\left|\mu_{\text {slow }}\right|}{\left|\mu_{\mathrm{nl}}\right|} \ll 1 \text {. }
$$

In systems that do not satisfy the condition of Eq. (11), it is still possible to use the reduced 1D model if one takes into account the contribution of $\mu_{\text {slow }}$ that plays a role of an additional potential.

The other approximation of the 1D effective model requires the nonlinear term $g|\varphi|^{2}$ to be small, such that the corresponding contribution to the chemical potential can be treated using perturbative methods and truncated after the first order contribution. As a result, the 1D model does not preserve the characteristic matter-wave collapse of the $3 \mathrm{D}$ GPE observed for $\mathcal{N}>2 k_{c}$ (with the critical value $k_{c}$ typically around $k_{c} \approx 0.627$ for isotropic transverse traps according to Ref. [31]). Other approaches are known to surpass these limitations [27,31], but our model should be suitable for typical experiments with low atomic densities.

\section{SPATIAL MODULATION OF THE NONLINEAR COEFFICIENT USING PINCHED TRANSVERSE POTENTIALS}

When the variation of the trapping frequencies is sufficiently slow along the longitudinal direction, the two degrees of freedom of the equivalent oscillator associated with the transverse confinement exchange energy adiabatically. For the particular choice proposed in Eq. (6), this process preserves the linear term of the expansion of the chemical potential in terms of $|\varphi|^{2}$, which corresponds to the effective 1D linear potential $V_{\text {eff }}$. The changes of the chemical potential occur only for higher order terms, associated with the effective nonlinearity. Indeed, as the imbalance between $\omega_{x}$ and $\omega_{y}$ increases, the equipotential lines of $V_{\text {ext }}(\mathbf{r})$ change from circumferences to ellipses, with increased area. This behavior, which resembles a pinching of the equipotential lines in the $x y$ plane, distorts the unperturbed ground-state wave function $\chi_{0}$ in a similar way, as illustrated in Fig. 1. Since the effective 1D nonlinearity scales with $\int\left|\chi_{0}\right|^{4} d x d y$, as the density becomes pinched and dispersed in the larger area, the nonlinear 

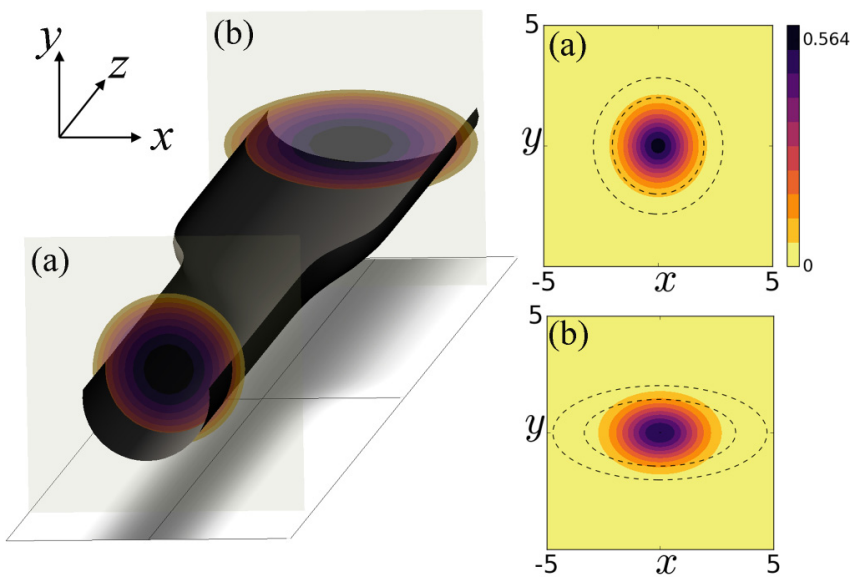

FIG. 1. Representation of the spatial structure of a pinch of the confinement potential described by Eq. (12). The black surface represents the lower half of an isosurface of $V_{\text {ext }}$ and illustrates the transition from a region with (a) $\omega_{x}=\omega_{y}=\bar{\omega}$ to (b) $\omega_{x}=\bar{\omega}-\Delta \omega$ and $\omega_{y}=\bar{\omega}+\Delta \omega$ for $\Delta \omega=0.4 \bar{\omega}$. In both insets equipotential lines (dashed lines) as well the local density of the transverse wave function $\chi_{0}$ are represented.

coefficient becomes weaker. For example, a relative change of the confinement frequencies $\Delta \omega / \bar{\omega}$ around $50 \%$ produces a relative change of $\tilde{g}_{\text {eff }}$ of about $13 \%$. Conversely, starting from an anisotropic transverse potential and decreasing the pinch on the potential towards the isotropic case increases the effective nonlinearity.

Compared with the standard technique of FRM, this pinching strategy presents two major advantages. First, it does not depend on the nature of the condensate and could even be employed in systems that do not support Feshbach resonances and where FRM is therefore not possible. Second, as it is independent of the confinement mechanism, it can be obtained both with magnetic and optical confinement. Experimentally, we anticipate that pinched magnetic potentials could be feasible by exploiting the geometry of the magnetic coils, using, for example, two rectangular coils [32] in anti-Helmholtz configuration with some misalignment. In the case of optical traps, one could superimpose two orthogonal light sheet potentials obtained by a time-averaged optical dipole potential using red-detuned laser fields $[33,34]$. In this setting, the strength of the dipole potential can be spatially modulated by controlling the velocity or intensity of the moving scanning laser [33]. Realizing such an experiment with optical confinement should be particularly relevant as it allows us to attain local variations of $V_{\text {eff }}$ and $g_{\text {eff }}$ at length scales much closer to the size of the condensate.

\section{SCATTERING OF MATTER-WAVE SOLITONS THROUGH A PINCH}

The results of the $1 \mathrm{D}$ effective model can be compared against the full 3D GPE, using a problem well established in the literature. In particular, we consider the scattering of matter wave solitons through a pinched potential characterized by

$$
\frac{\Delta \omega(z)}{\bar{\omega}}=\frac{\eta}{2}[1+\tanh (\gamma z)]
$$

corresponding to a model for a smooth transition between two sections with constant yet distinct nonlinear coefficient, more precisely from $g_{\text {eff }}(z \rightarrow-\infty)=1$ to $g_{\text {eff }}(z \rightarrow+\infty)=$ $\sqrt{1-\eta^{2}}$. The parameters $\eta$ and $\gamma$ characterize the strength and smoothness of the pinch, respectively. For $z \rightarrow \pm \infty, \omega_{x}$, $\omega_{y}$, and $\tilde{g}_{\text {eff }}(z)$ are approximately constant and Eq. (7) supports soliton solutions that can be either of the bright or dark type depending on if the nonlinearity is positive or negative. Here, we choose to consider the case of bright solitons and to leave dark solitons for a future work. For a region of constant nonlinearity $g_{0}$, a bright soliton solution can be expressed as [35]

$$
\varphi(z, t)=\frac{a}{g_{0}} \operatorname{sech}[a(z-v t)] \exp \left[i v z-i\left(a^{2}-v^{2}\right) t / 2\right],
$$

where $a$ and $v$ are the amplitude and the velocity of the soliton, respectively. The analysis of the scattering of solitons through the region near $z=0$ was investigated in terms of their transmission or reflection using numerical simulations based on the pseudospectral split-step Fourier method to integrate both the full 3D and 1D GPEs [respectively, Eqs. (1) and (7)] with initial condition given by Eq. (13) and corresponding to an initial amplitude $a_{i}$ and velocity $v_{i}$. Also, we choose the initial position of the center of the soliton $z_{0} \ll 0$, such that the soliton is initially far away from the pinch. The numerical simulations consider pinch strengths $\eta$ in the range $[0,0.6]$, soliton amplitudes within [0.2,0.4], as well as velocities in the interval [0.01,0.07]. Under these conditions, the requirement described in Eq. (11) is satisfied for $\gamma \ll 2 a$, which leads to the choice of $\gamma=0.1$.

Typical results of the 3D simulations for the scattering process are presented in Figs. 2 and 3 and they show that matter-wave soliton exhibits two distinct behaviors depending on the strength of the pinch $\eta$. For weak pinches $\left(\eta<\eta_{c}\right)$, the soliton is totally transmitted with a decrease in the
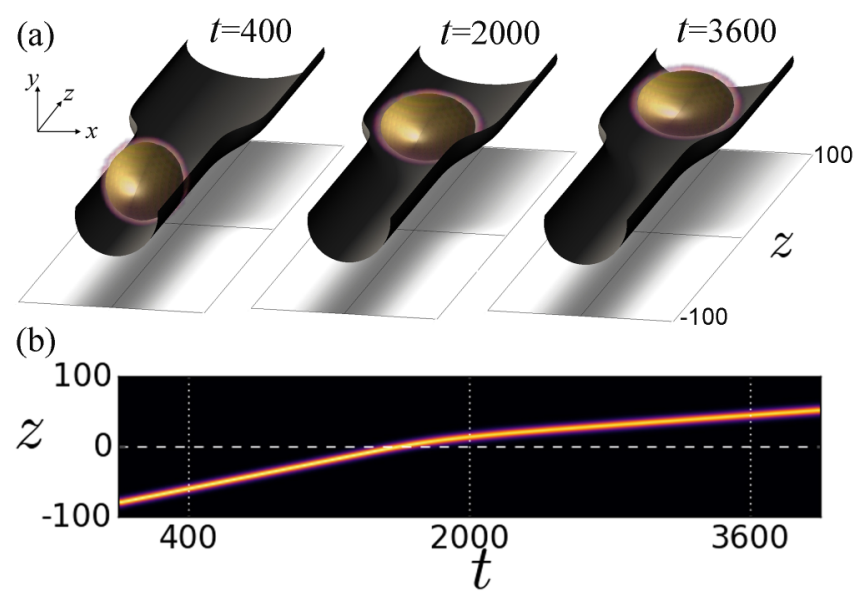

FIG. 2. Numerical simulations for the evolution of a bright soliton with initial amplitude $a_{i}=0.2$ and velocity $v_{i}=0.05$ scattered by a pinching of strength $\eta=0.4$ centered at $z=0$. (a) Timeframes of the transmission of the soliton. The black isosurface corresponds to an isosurface of $V_{\text {ext }}$, and the yellow isosurface to the wave-function density $|\Psi|^{2}$. (b) Spatiotemporal contour plots of the density $|\varphi|^{2}$ obtained after integration of the transverse dependence. Animation of the simulation is included in the Supplemental Material [36]. 


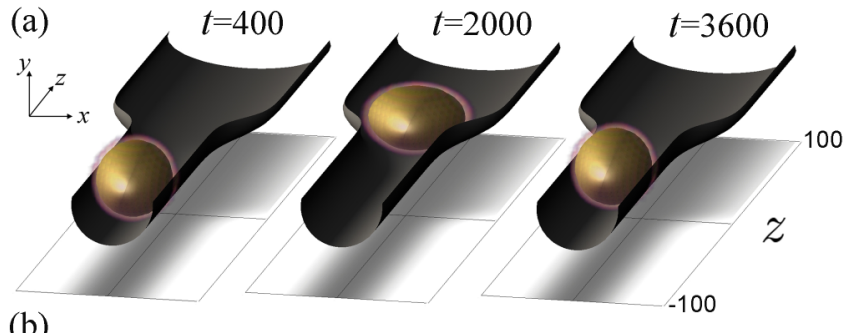

(b)

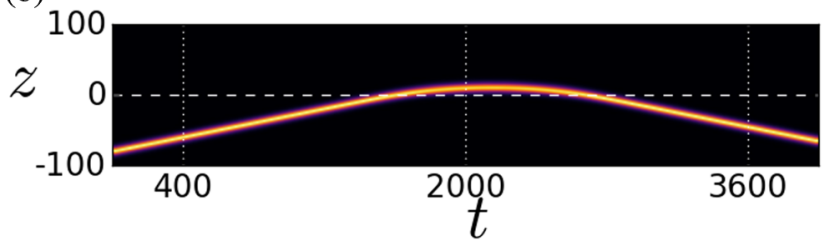

FIG. 3. Same as Fig. 2 for a soliton with initial amplitude $a_{i}=0.2$ and velocity $v_{i}=0.05$ scattered by a pinching of strength $\eta=0.5$ centered at $z=0$. Animation of the simulation is included in the Supplemental Material [36].

velocity, whereas for strong pinches $\left(\eta>\eta_{\mathrm{c}}\right)$ it undergoes total reflection. Indeed, numerical calculations of the transmission coefficient $\mathcal{T} \equiv \int_{0}^{+\infty}|\varphi|^{2} d z / \mathcal{N}$ show a steplike dependence on the pinch strength $\eta$ (see Fig. 4). Simulations using the 1D effective model yield similar results, with an error below $5 \%$ when compared to the full 3D simulations, thus confirming the validity of our model and that the pinching of the transverse confinement potential is equivalent to a spatial modulation of the nonlinearity.

The critical value of the pinch strength $\eta_{c}$ can be estimated analytically assuming that the asymptotic initial and final states (respectively for $t \rightarrow-\infty$ and $t \rightarrow+\infty$ ) are localized far away from $z=0$ (i.e., where the nonlinear coefficient $g_{\text {eff }}$ is constant) and are of the form of Eq. (13). Then, using the conservation laws of Eq. (7), namely of $\mathcal{N}$ and $\mathcal{H}$, the final amplitude $a_{f}$ and velocity $v_{f}$ (for $t \rightarrow+\infty$ ) for a totally
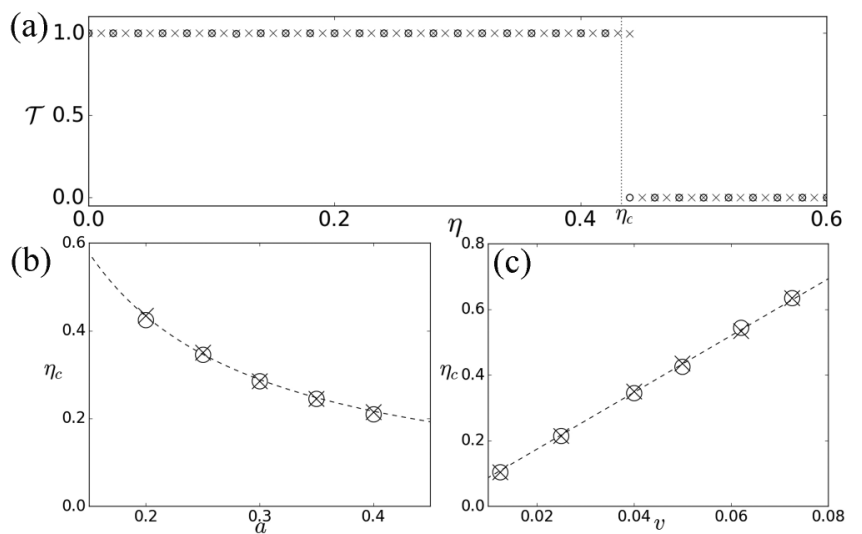

FIG. 4. Numerical results of the 3D GPE (circles) and of the 1D effective model (crosses) for the outcome of the scattering process of a bright soliton with initial amplitude $a_{i}=0.2$ and velocity $v_{i}=0.05$, in comparison with the theoretical prediction (dashed lines). (a) Transmission coefficient in function of the pinch strength. Dependence of the critical value $\eta_{c}$ on the (b) initial amplitude and (c) initial velocity. reflected soliton are

$$
a_{f}=a_{i}, \quad v_{f}=-v_{i},
$$

while for totally transmitted solitons they are

$$
a_{f}=a_{i} \sqrt{1-\eta^{2}}, \quad v_{f}^{2}=v_{i}^{2}-\frac{a_{i}^{2} \eta^{2}}{3} .
$$

For physical solutions, the right-hand side of the velocity law should be positive, which is only allowed for a pinch strength below the critical value $\eta<\eta_{c}=\sqrt{3} v / a$. Figure 4 shows a good agreement between the simulation results and the analytical predictions for $\eta_{c}$ as a function of the initial amplitude and the velocity for soliton amplitudes below the collapse threshold (well within the validity of the model). These results are qualitatively similar to those described in the literature for the scattering of solitons through combined linear and nonlinear inhomogeneities, as well as pure nonlinear inhomogeneities, which in some cases also predict regimes of total transmission and total reflection $[15,19]$. Still, in our case it is noteworthy that transition between the two regimes is steplike at a critical parameter. This behavior arises from the nonlinear inhomogeneity exclusively, since no linear inhomogeneity is present (at least from the point of view of the 1D model). Also, as the nonlinearity produced by the pinch varies at scales larger than the soliton width, it can evolve almost adiabatically, and preserve the soliton-like shape [16,19]. This steplike transition between total transmission and reflection could in principle be used to develop a tunable pinch valve or filter. A richer phenomenology is predicted to occur when one considers not only the scattering of bright solitons by a spatial modulation of the nonlinearity but also the effect of additional linear potentials. Examples include the observation of the enhancement of transmission through a potential barrier in the presence of nonlinear inhomogeneities $[15,16]$ and the population of bound states in potential wells [37]. Such effects could be explored under the formalism of the pinching of the trapping potential by selecting other forms for the variation of the $\omega_{x}(z)$ and $\omega_{y}(z)$ transverse trapping frequencies.

\section{CONCLUSIONS}

Through the development of an effective model, we have presented how an anisotropic variation of the transverse confinement potential can be used to control independently the effective linear potential and effective nonlinearity along the longitudinal direction of a quasi-1D Bose-Einstein condensate (BEC). We have also demonstrated that the pinching of the potential allows us to produce pure modulations of the nonlinearity, unlike other approaches [27,28]. This method can be realized for any kind of BEC, using either magnetic or optical confinement strategies, and could be specially relevant to study the dynamics of condensates when the use of the Feshbach resonance is inadequate. Using numerical simulations, we have investigated the scattering of bright solitons through a pinched potential, finding excellent agreement between the results obtained for the reduced $1 \mathrm{D}$ and the complete 3D Gross-Pitaevskii equation. By considering the parameters for a condensate made of ${ }^{7} \mathrm{Li}$ atoms [38], the results obtained in terms of dimensionless variables can easily provide quantitative estimations of the scales involved. Considering a 
typical scattering length of $a_{s}=-1.45 \mathrm{~nm}$ and a transverse confining frequency $\bar{\omega}=2 \pi \times 100 \mathrm{~Hz}$, the soliton amplitude $a=0.2$ corresponds to a condensate of about $N_{a} \approx 500$ atoms with a typical size of $L_{c} \approx \sqrt{\hbar / \bar{\omega} m} / a \approx 20 \mu \mathrm{m}$. Under these conditions, a pinching of the potential of the form suggested in Sec. IV could be realized in a spatial scale $L_{p} \approx \sqrt{\hbar / \bar{\omega} m} / \gamma \approx$ $40 \mu \mathrm{m}$, which is still comparable to the size of the soliton.

Finally, it is also relevant to mention that despite our focus on the case of bright solitons, which results in atomic condensates with attractive interactions, the 1D effective model is independent of the sign of the scattering length and can be extended to explore nonlinear phenomena in systems with repulsive interactions, such as the dynamics of dark matter waves. We hope that this confinement pinch mechanism stimulates experimental proposals not only to test it but also to explore and control matter-wave dynamics in BECs.

\section{ACKNOWLEDGMENTS}

The authors thank V. Brazhnyy for discussions and comments on the manuscript. This work is financed by the ERDF (European Regional Development Fund) through the Operational Programme for Competitiveness and Internationalisation - COMPETE 2020 Programme, and by national funds through the FCT (Fundacao para a Ciencia e a Tecnologia, Portuguese Foundation for Science and Technology) within Project No. POCI-01-0145-FEDER-006961 and Grant No. SFRH/BD/105486/2014.
[1] Y. V. Kartashov, B. A. Malomed, and L. Torner, Rev. Mod. Phys. 83, 247 (2011).

[2] P. G. Kevrekidis, D. J. Frantzeskakis, and R. CarreteroGonzález, Emergent Nonlinear Phenomena in Bose-Einstein Condensates: Theory and Experiment (Springer Science \& Business Media, Berlin, 2007), Vol. 45.

[3] H. Saito and M. Ueda, Phys. Rev. Lett. 90, 040403 (2003).

[4] M. Centurion, M. A. Porter, P. G. Kevrekidis, and D. Psaltis, Phys. Rev. Lett. 97, 033903 (2006).

[5] M. Centurion, M. A. Porter, Y. Pu, P. G. Kevrekidis, D. J. Frantzeskakis, and D. Psaltis, Phys. Rev. Lett. 97, 234101 (2006).

[6] P. G. Kevrekidis, G. Theocharis, D. J. Frantzeskakis, and B. A. Malomed, Phys. Rev. Lett. 90, 230401 (2003).

[7] D. E. Pelinovsky, P. G. Kevrekidis, and D. J. Frantzeskakis, Phys. Rev. Lett. 91, 240201 (2003).

[8] Z. X. Liang, Z. D. Zhang, and W. M. Liu, Phys. Rev. Lett. 94, 050402 (2005).

[9] V. A. Brazhnyi and V. V. Konotop, Phys. Rev. A 72, 033615 (2005).

[10] V. M. Pérez-García, V. V. Konotop, and V. A. Brazhnyi, Phys. Rev. Lett. 92, 220403 (2004).

[11] F. K. Abdullaev and M. Salerno, J. Phys. B 36, 2851 (2003).

[12] M. I. Rodas-Verde, H. Michinel, and V. M. Pérez-García, Phys. Rev. Lett. 95, 153903 (2005).

[13] A. V. Carpentier, H. Michinel, M. I. Rodas-Verde, and V. M. Pérez-García, Phys. Rev. A 74, 013619 (2006).

[14] H. Michinel, A. Paredes, M. M. Valado, and D. Feijoo, Phys. Rev. A 86, 013620 (2012).

[15] J. Garnier and F. K. Abdullaev, Phys. Rev. A 74, 013604 (2006).

[16] P. Niarchou, G. Theocharis, P. G. Kevrekidis, P. Schmelcher, and D. J. Frantzeskakis, Phys. Rev. A 76, 023615 (2007).

[17] A. Balaž, R. Paun, A. I. Nicolin, S. Balasubramanian, and R. Ramaswamy, Phys. Rev. A 89, 023609 (2014).

[18] G. Theocharis, P. Schmelcher, P. G. Kevrekidis, and D. J. Frantzeskakis, Phys. Rev. A 72, 033614 (2005).

[19] G. Theocharis, P. Schmelcher, P. G. Kevrekidis, and D. J. Frantzeskakis, Phys. Rev. A 74, 053614 (2006).
[20] M. Salerno, V. V. Konotop, and Y. V. Bludov, Phys. Rev. Lett. 101, 030405 (2008).

[21] R. Balbinot, A. Fabbri, S. Fagnocchi, A. Recati, and I. Carusotto, Phys. Rev. A 78, 021603 (2008).

[22] I. Carusotto, S. Fagnocchi, A. Recati, R. Balbinot, and A. Fabbri, New J. Phys. 10, 103001 (2008).

[23] J. Steinhauer, Nat. Phys. 12, 959 (2016).

[24] S. Inouye, M. Andrews, J. Stenger, H.-J. Miesner, D. StamperKurn, and W. Ketterle, Nature (London) 392, 151 (1998).

[25] M. Theis, G. Thalhammer, K. Winkler, M. Hellwig, G. Ruff, R. Grimm, and J. H. Denschlag, Phys. Rev. Lett. 93, 123001 (2004).

[26] R. Yamazaki, S. Taie, S. Sugawa, and Y. Takahashi, Phys. Rev. Lett. 105, 050405 (2010).

[27] S. D. Nicola, B. A. Malomed, and R. Fedele, Phys. Lett. A 360, 164 (2006).

[28] L. Salasnich, A. Cetoli, B. A. Malomed, F. Toigo, and L. Reatto, Phys. Rev. A 76, 013623 (2007).

[29] V. M. Pérez-García, H. Michinel, and H. Herrero, Phys. Rev. A 57, 3837 (1998).

[30] A. Gammal, L. Tomio, and T. Frederico, Phys. Rev. A 66, 043619 (2002).

[31] L. Khaykovich and B. A. Malomed, Phys. Rev. A 74, 023607 (2006).

[32] Y.-W. Lin, H.-C. Chou, P. P. Dwivedi, Y.-C. Chen, and A. Y. Ite, Opt. Express 16, 3753 (2008).

[33] S. K. Schnelle, E. D. van Ooijen, M. J. Davis, N. R. Heckenberg, and H. Rubinsztein-Dunlop, Opt. Express 16, 1405 (2008).

[34] T. A. Bell, J. A. Glidden, L. Humbert, M. W. Bromley, S. A. Haine, M. J. Davis, T. W. Neely, M. A. Baker, and H. Rubinsztein-Dunlop, New J. Phys. 18, 035003 (2016).

[35] V. E. Zakharov and A. B. Shabat, Zh. Eksp. Teor. Fiz. 61, 118 (1971) [Sov. Phys. JETP 34, 62 (1972)].

[36] See Supplemental Material at http://link.aps.org/supplemental/ 10.1103/PhysRevA.94.063602 for animations of the numerical simulations of the scattering process of solitons through a pinch.

[37] F. Damon, B. Georgeot, and D. Guéry-Odelin, Europhys. Lett. 115, 20010 (2016).

[38] C. Sackett, C. Bradley, M. Welling, and R. G. Hulet, Appl. Phys. B 65, 433 (1997). 